\title{
Two-mode single-atom laser as a source of entangled light
}

\author{
M. Kiffner, ${ }^{1, *}$ M. S. Zubairy, ${ }^{1,2,3, \dagger}$ J. Evers, ${ }^{1, \dagger}$ and C. H. Keitel ${ }^{1, \S}$ \\ ${ }^{1}$ Max-Planck-Institut für Kernphysik, Saupfercheckweg 1, 69117 Heidelberg, Germany \\ ${ }^{2}$ Institute for Quantum Studies and Department of Physics, Texas A\&M University, College Station, Texas 77843, USA \\ ${ }^{3}$ Texas A\&M University at Qatar, Education City, P.O. Box 23874, Doha, Qatar
}

(Received 22 December 2006; revised manuscript received 25 January 2007; published 28 March 2007)

\begin{abstract}
A two-mode single-atom laser is considered, with the aim of generating entanglement in macroscopic light. Two transitions in the four-level gain medium atom independently interact with the two cavity modes, while two other transitions are driven by control laser fields. Atomic relaxation as well as cavity losses are taken into account. We show that this system is a source of macroscopic entangled light over a wide range of control parameters and initial states of the cavity field.
\end{abstract}

DOI: 10.1103/PhysRevA.75.033816

PACS number(s): 42.50.Dv, 03.67.Mn, 42.50.Pq

\section{INTRODUCTION}

Quantum entanglement is known to be the key resource in many applications of quantum information and quantum computing [1]. These phenomena range from quantum teleportation [2,3] and quantum cryptography [4] to quantum implementation of Shor's algorithm [5] and quantum search [6]. It is therefore not surprising that there has been a great deal of interest in the generation and measurement of entanglement in recent years.

Entangled states have been considered traditionally between individual qubits. However, it has been shown that continuous-variable entanglement can offer an advantage in some situations in quantum-information science [7]. One reason for this is that continuous-variable entanglement often can be prepared unconditionally, whereas the preparation of discrete entanglement usually relies on an event selection via coincidence measurements. The classic scheme for the generation of continuous-variable entanglement is parametric down-conversion. Starting with the first demonstration by Ou et al. [8], the generation of entanglement in such systems has been achieved in several experiments [7]. It still remains, however, a challenge to generate entanglement in macroscopic light rather than on the few-photon level. Promising candidates for the generation of macroscopic light entanglement are optical amplifiers [9-13]. For example, it was shown recently that a two-mode correlated spontaneous emission laser $[14,15]$ can lead to two-mode entanglement even when the average photon number in the field modes is very large $[12,13]$. In this setup, the gain medium can be thought of as a stream of suitably prepared atoms.

From a conceptual point of view, a much simpler system relates to a single-atom laser, where the gain medium is replaced by a single trapped atom. Such a laser has recently been experimentally demonstrated by Kimble and coworkers [16], where a single atom interacts with a single cavity mode. Thus the interesting question arises as to

\footnotetext{
*Electronic address: martin.kiffner@mpi-hd.mpg.de

${ }^{\dagger}$ Electronic address: zubairy@physics.tamu.edu

*Electronic address: joerg.evers@ mpi-hd.mpg.de

${ }^{\S}$ Electronic address: keitel@mpi-hd.mpg.de
}

whether a two-mode generalization of the single-atom laser also enables one to generate entanglement in macroscopic light.

Therefore, here we consider a single atom that interacts with two quantized modes of a doubly resonant cavity via two lasing transitions. In our model, the atomic level scheme is based on the single-atom laser experiment performed by Kimble and co-workers [16], where dipole transitions between four hyperfine levels of atomic cesium were considered. In contrast to their experiment, we do not work in the strong-coupling regime since we are interested in the generation of large photon numbers. We show that, under certain realizable conditions, a two-mode single-atom laser can serve as a source of macroscopic entangled light. Macroscopic entanglement can be achieved over a wide range of control parameters and initial states of the cavity field.

An important technical question in the generation of continuous-variable entanglement in quantum optical systems is the way such entanglement can be measured experimentally. This has been a hotly discussed subject in recent years. Several inequalities involving the correlation of the field operators have been derived that are based on the separability condition of the field modes [17-24]. A violation of these inequalities provides evidence of entanglement. These inequalities can, in general, provide only a sufficient condition for entanglement and only, in some very specific instances, lead to sufficient and necessary conditions for entanglement. In this paper we use an inequality based on quadrature measurement of the field variables for the test of entanglement.

\section{MASTER EQUATION FOR THE DENSITY OPERATOR OF THE CAVITY MODES}

We consider a single four-level atom trapped in a doubly resonant cavity (see Fig. 1). The atom interacts with two (nondegenerate) cavity modes and two classical laser fields. The intensities and frequencies of the two laser fields can be adjusted independently. The aim of this section is to derive an equation of motion for the reduced density operator $\varrho_{\mathrm{F}}$ of the two cavity modes.

We begin with a detailed description of the system shown in Fig. 1 . The first cavity mode with frequency $\nu_{1}$ couples to 


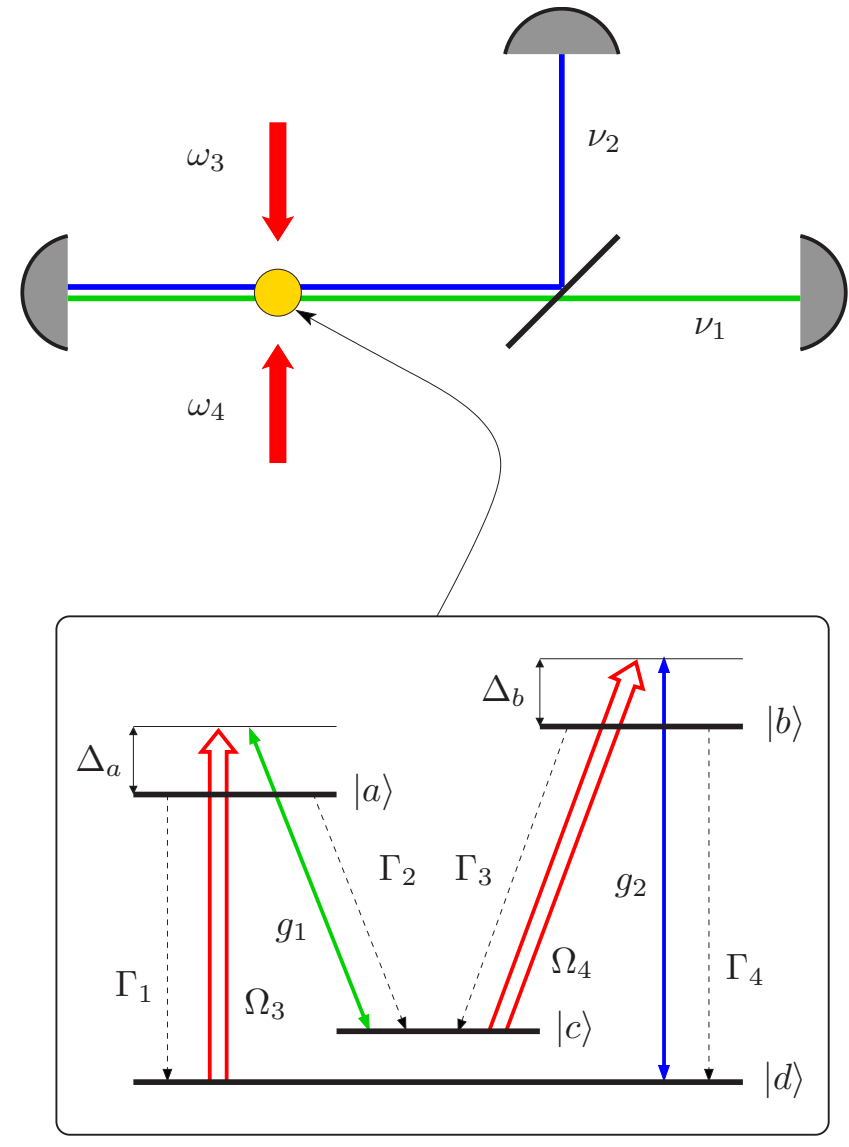

FIG. 1. (Color online) A single four-level atom is trapped in a doubly resonant cavity and interacts with two cavity modes and two classical laser fields. The inset shows the atomic level scheme. The laser field with frequency $\omega_{3}$ and Rabi frequency $\Omega_{3}$ couples to the $|a\rangle \leftrightarrow|d\rangle$ transition, and the cavity mode with frequency $\nu_{1}$ and coupling constant $g_{1}$ interacts with the $|a\rangle \leftrightarrow|c\rangle$ transition. $\Delta_{a}$ is the detuning of the fields $\Omega_{3}$ and $g_{1}$ from state $|a\rangle$. The laser field with frequency $\omega_{4}$ and Rabi frequency $\Omega_{4}$ drives the $|b\rangle \leftrightarrow|c\rangle$ transition, and the second cavity mode with frequency $\nu_{2}$ and coupling constant $g_{2}$ interacts with the $|b\rangle \leftrightarrow|d\rangle$ transition. $\Delta_{b}$ is the detuning of the fields $\Omega_{4}$ and $g_{2}$ from state $|b\rangle$. Spontaneous emission is denoted by dashed arrows, and the parameters $\Gamma_{i}$ are the decay rates of the various transitions.

the atomic transition $|a\rangle \leftrightarrow|c\rangle$, and the second mode with frequency $\nu_{2}$ interacts with the atom on the $|b\rangle \leftrightarrow|d\rangle$ transition. In the rotating-wave approximation, the interaction of the atom with the cavity modes is described by the Hamiltonian

$$
H_{\mathrm{C}}=\hbar g_{1} a_{1}|a\rangle\left\langle c\left|+\hbar g_{2} a_{2}\right| b\right\rangle\langle d|+\text { H.c. }
$$

Here $a_{j}\left(a_{j}^{\dagger}\right)$ is the annihilation (creation) operator of the cavity mode with frequency $\nu_{j}$ and coupling constant $g_{j}(j$ $\in\{1,2\})$. The detuning of the first cavity mode from the $|a\rangle \leftrightarrow|c\rangle$ transition is denoted by $\Delta_{1}$, and $\Delta_{2}$ is the detuning of the second mode from the $|b\rangle \leftrightarrow|d\rangle$ transition,

$$
\Delta_{1}=\nu_{1}-\omega_{a c}, \quad \Delta_{2}=\nu_{2}-\omega_{b d} .
$$

The resonance frequencies on the $|a\rangle \leftrightarrow|c\rangle$ and $|b\rangle \leftrightarrow|d\rangle$ transitions have been labeled by $\omega_{a c}$ and $\omega_{b d}$, respectively. In addition, the atom interacts with two classical laser fields. The first laser field with frequency $\omega_{3}$ and Rabi frequency $\Omega_{3}$ couples to the $|a\rangle \leftrightarrow|d\rangle$ transition, and the second field with frequency $\omega_{4}$ and Rabi frequency $\Omega_{4}$ coherently drives the $|b\rangle \leftrightarrow|c\rangle$ transition. In the rotating-wave approximation, the atom-laser interaction reads

$$
H_{\mathrm{L}}=-\hbar \Omega_{3}|a\rangle\left\langle d\left|e^{-i \omega_{3} t}-\hbar \Omega_{4}\right| b\right\rangle\langle c| e^{-i \omega_{4} t}+\text { H.c. }
$$

Note that the Rabi frequencies $\Omega_{3}=\left|\Omega_{3}\right| \exp \left(i \phi_{3}\right)$ and $\Omega_{4}$ $=\left|\Omega_{4}\right| \exp \left(i \phi_{4}\right)$ are complex numbers, and $\phi_{3}$ and $\phi_{4}$ are determined by the phase of the laser fields. The detunings of the laser fields from the corresponding atomic transitions are

$$
\Delta_{3}=\omega_{3}-\omega_{a d}, \quad \Delta_{4}=\omega_{4}-\omega_{b c},
$$

where $\omega_{a d}$ and $\omega_{b c}$ are the resonance frequencies on the $|a\rangle \leftrightarrow|d\rangle$ and $|b\rangle \leftrightarrow|c\rangle$ transitions, respectively.

The free time evolution of the cavity modes is governed by

$$
H_{\mathrm{R}}=\hbar \nu_{1} a_{1}^{\dagger} a_{1}+\hbar \nu_{2} a_{2}^{\dagger} a_{2},
$$

and $H_{\mathrm{A}}$ is the free Hamiltonian of the atomic degrees of freedom,

$$
H_{\mathrm{A}}=\hbar \omega_{a}|a\rangle\left\langle a\left|+\hbar \omega_{b}\right| b\right\rangle\left\langle b\left|+\hbar \omega_{c}\right| c\right\rangle\left\langle c\left|+\hbar \omega_{d}\right| d\right\rangle\langle d| .
$$

With these definitions, we arrive at the master equation for the combined system of the atomic degrees of freedom and the two cavity modes,

$$
\dot{\varrho}=-\frac{i}{\hbar}\left[H_{\mathrm{R}}+H_{\mathrm{A}}+H_{\mathrm{L}}+H_{\mathrm{C}}, \varrho\right]+\mathcal{L}_{\gamma} \varrho .
$$

The last term in Eq. (7) accounts for spontaneous emission and is given by

$$
\mathcal{L}_{\gamma} \varrho=-\frac{1}{2} \sum_{i=1}^{4} \Gamma_{i}\left(S_{i}^{+} S_{i}^{-} \varrho+\varrho S_{i}^{+} S_{i}^{-}-2 S_{i}^{-} \varrho S_{i}^{+}\right),
$$

where the atomic transition operators are defined as

$$
\begin{gathered}
S_{1}^{+}=|a\rangle\left\langle d\left|, \quad S_{2}^{+}=\right| a\right\rangle\langle c|, \\
S_{3}^{+}=|b\rangle\left\langle c\left|, \quad S_{4}^{+}=\right| b\right\rangle\langle d|, \quad S_{i}^{-}=\left(S_{i}^{+}\right)^{\dagger} .
\end{gathered}
$$

The parameters $\Gamma_{i}$ are the decay rates of the various atomic transitions (see Fig. 1).

In the next step, we derive from Eq. (7) the master equation for the density operator $\varrho_{\mathrm{F}}$ of the cavity modes,

$$
\varrho_{\mathrm{F}}=\operatorname{Tr}_{\mathrm{A}} \varrho=\varrho_{a a}+\varrho_{b b}+\varrho_{c c}+\varrho_{d d},
$$

and $\varrho_{\nu \nu}$ denotes $\langle\nu|\varrho| \nu\rangle$. To this end, we apply a unitary transformation $W=W_{\mathrm{R}} \otimes W_{\mathrm{A}}$ to Eq. (7), where $W_{\mathrm{R}}$ $=\exp \left[i H_{\mathrm{R}} t / \hbar\right]$ acts only on the cavity modes, and

$$
W_{\mathrm{A}}=\exp \left[i\left(H_{\mathrm{A}}+\hbar \Delta_{3}|a\rangle\left\langle a\left|+\hbar \Delta_{4}\right| b\right\rangle\langle b|\right) t / \hbar\right]
$$

acts only on the atomic degrees of freedom. As indicated in Fig. 1, we assume that the condition of two-photon resonance is satisfied, i.e., 


$$
\Delta_{a}=\Delta_{1}=\Delta_{3}, \quad \Delta_{b}=\Delta_{2}=\Delta_{4} .
$$

The density operator in the new frame is denoted by $\widetilde{\varrho}$ $=W \varrho W^{\dagger}$ and obeys the equation of motion

$$
\dot{\widetilde{\varrho}}=-\frac{i}{\hbar}\left[H_{0}+H_{\mathrm{C}}, \widetilde{\varrho}\right]+\mathcal{L}_{\gamma} \widetilde{\varrho},
$$

where

$H_{0}=-\hbar \Delta_{a}|a\rangle\left\langle a\left|-\hbar \Delta_{b}\right| b\right\rangle\langle b|-\hbar\left(\Omega_{3}|a\rangle\left\langle d\left|+\Omega_{4}\right| b\right\rangle\langle c|+\right.$ H.c. $)$.

The two-photon condition Eq. (12) ensures that the Hamiltonian $H_{0}+H_{\mathrm{C}}$ in Eq. (13) is time independent. The master equation for the transformed density operator $\widetilde{\varrho}_{F}$ of the cavity modes is obtained if we trace over the atomic degrees of freedom in Eq. (13),

$$
\dot{\widetilde{\varrho}}_{\mathrm{F}}=-i g_{1}\left[a_{1}^{\dagger}, \widetilde{\varrho}_{a c}\right]-i g_{2}\left[a_{2}^{\dagger}, \widetilde{\varrho}_{b d}\right]+\text { H.c. }
$$

In order to eliminate the coherences $\widetilde{\varrho}_{a c}$ and $\widetilde{\varrho}_{b d}$ from Eq. (15), we apply the standard methods of laser theory (see, e.g., Chapter 14 in [25]). We restrict the analysis to the linear theory and solve Eq. (13) to first order in the coupling constants $g_{1}$ and $g_{2}$. To this end, we expand the density operator $\widetilde{\varrho}_{\mathrm{F}}$ in Eq. (13) as $\widetilde{\varrho}=\varrho_{0}+\varrho_{C}$ and retain only terms up to first order with respect to $H_{\mathrm{C}}$. This procedure yields two uncoupled equations for $\varrho_{0}$ and $\varrho_{C}$,

$$
\dot{\varrho}_{0}=\mathcal{L}_{0} \varrho_{0}
$$

$$
\dot{\varrho_{C}}=\mathcal{L}_{0} \varrho_{C}-\frac{i}{\hbar}\left[H_{\mathrm{C}}, \varrho_{0}\right]
$$

and the superoperator $\mathcal{L}_{0}$ is defined as

$$
\mathcal{L}_{0}(\cdot)=-\frac{i}{\hbar}\left[H_{0}, \cdot\right]+\mathcal{L}_{\gamma}(\cdot) .
$$

Here the centered dot denotes the position of the argument of $\mathcal{L}_{0}$. The zeroth-order equation (16) describes the interaction of the atom with the classical laser fields to all orders, and Eq. (17) is the first-order equation. The steady-state solution for $\widetilde{\varrho}_{a c}$ and $\widetilde{\varrho}_{b d}$ can be obtained if the steady-state solution for $\varrho_{0}$ is plugged in Eq. (17). We find

$$
\begin{aligned}
& i g_{1} \widetilde{\varrho}_{a c}=\alpha_{11} a_{1} \widetilde{\varrho}_{\mathrm{F}}+\alpha_{12} a_{2}^{\dagger} \widetilde{\varrho}_{\mathrm{F}}+\beta_{11} \widetilde{\varrho}_{\mathrm{F}} a_{1}+\beta_{12} \widetilde{\varrho}_{\mathrm{F}} a_{2}^{\dagger}, \\
& i g_{2} \widetilde{\varrho}_{b d}=\alpha_{22} a_{2} \widetilde{\varrho}_{\mathrm{F}}+\alpha_{21} a_{1}^{\dagger} \widetilde{\varrho}_{\mathrm{F}}+\beta_{22} \widetilde{\varrho}_{\mathrm{F}} a_{2}+\beta_{21} \widetilde{\varrho}_{\mathrm{F}} a_{1}^{\dagger},
\end{aligned}
$$

and the coefficients $\alpha_{i j}$ and $\beta_{i j}$ are defined in Appendix A. Next we substitute Eq. (19) in Eq. (15) to obtain the equation of motion for $\widetilde{\varrho}_{\mathrm{F}}$. Finally, we transform $\widetilde{\varrho}_{\mathrm{F}}$ back with respect to $W_{R}$ and obtain the equation of motion for the density operator $\varrho_{\mathrm{F}}$ of the cavity modes,

$$
\begin{aligned}
\dot{\varrho}_{\mathrm{F}}= & -i \nu_{1}\left[a_{1}^{\dagger} a_{1}, \varrho_{\mathrm{F}}\right]-i \nu_{2}\left[a_{2}^{\dagger} a_{2}, \varrho_{\mathrm{F}}\right]-\left[\alpha_{11} a_{1}^{\dagger} a_{1} \varrho_{\mathrm{F}}+\alpha_{11}^{*} \varrho_{\mathrm{F}} a_{1}^{\dagger} a_{1}-\left(\alpha_{11}+\alpha_{11}^{*}\right) a_{1} \varrho_{\mathrm{F}} a_{1}^{\dagger}-\beta_{11}^{*} a_{1} a_{1}^{\dagger} \varrho_{\mathrm{F}}-\beta_{11} \varrho_{\mathrm{F}} a_{1} a_{1}^{\dagger}\right. \\
& \left.+\left(\beta_{11}+\beta_{11}^{*}\right) a_{1}^{\dagger} \varrho_{\mathrm{F}} a_{1}\right]-\left[\alpha_{22} a_{2}^{\dagger} a_{2} \varrho_{\mathrm{F}}+\alpha_{22}^{*} \varrho_{\mathrm{F}} a_{2}^{\dagger} a_{2}-\left(\alpha_{22}+\alpha_{22}^{*}\right) a_{2} \varrho_{\mathrm{F}} a_{2}^{\dagger}-\beta_{22}^{*} a_{2} a_{2}^{\dagger} \varrho_{\mathrm{F}}-\beta_{22} \varrho_{\mathrm{F}} a_{2} a_{2}^{\dagger}+\left(\beta_{22}+\beta_{22}^{*}\right) a_{2}^{\dagger} \varrho_{\mathrm{F}} a_{2}\right] \\
& -\left[\left(\alpha_{12}+\alpha_{21}\right) a_{1}^{\dagger} a_{2}^{\dagger} \varrho_{\mathrm{F}}-\left(\beta_{12}+\beta_{21}\right) \varrho_{\mathrm{F}} a_{1}^{\dagger} a_{2}^{\dagger}-\left(\alpha_{21}-\beta_{12}\right) a_{1}^{\dagger} \varrho_{\mathrm{F}} a_{2}^{\dagger}-\left(\alpha_{12}-\beta_{21}\right) a_{2}^{\dagger} \varrho_{\mathrm{F}} a_{1}^{\dagger}\right] \exp \left[-i\left(\nu_{1}+\nu_{2}\right) t\right] \\
& -\left[\left(\alpha_{12}^{*}+\alpha_{21}^{*}\right) \varrho_{\mathrm{F}} a_{1} a_{2}-\left(\beta_{12}^{*}+\beta_{21}^{*}\right) a_{1} a_{2} \varrho_{\mathrm{F}}-\left(\alpha_{21}^{*}-\beta_{12}^{*}\right) a_{2} \varrho_{\mathrm{F}} a_{1}-\left(\alpha_{12}^{*}-\beta_{21}^{*}\right) a_{1} \varrho_{\mathrm{F}} a_{2}\right] \exp \left[i\left(\nu_{1}+\nu_{2}\right) t\right] \\
& -\kappa_{1}\left(a_{1}^{\dagger} a_{1} \varrho_{\mathrm{F}}+\varrho_{\mathrm{F}} a_{1}^{\dagger} a_{1}-2 a_{1} \varrho_{\mathrm{F}} a_{1}^{\dagger}\right)-\kappa_{2}\left(a_{2}^{\dagger} a_{2} \varrho_{\mathrm{F}}+\varrho_{\mathrm{F}} a_{2}^{\dagger} a_{2}-2 a_{2} \varrho_{\mathrm{F}} a_{2}^{\dagger}\right) .
\end{aligned}
$$

In the last line of Eq. (20), we included the damping of the cavity field. The damping constants of the cavity modes are denoted by $\kappa_{1}$ and $\kappa_{2}$, respectively.

In the master equation (20), the two classical laser fields are taken into account to all orders in the Rabi frequencies $\Omega_{3}$ and $\Omega_{4}$. On the contrary, the two quantum fields inside the cavity are only treated to second order in the coupling constants $g_{1}$ and $g_{2}$. This approximation means that we ignore saturation effects and operate in the regime of linear amplification. It is justified if the Rabi frequencies associated with the quantum fields are small as compared to other system parameters which dominate the time evolution.

\section{ENTANGLEMENT OF THE CAVITY FIELD}

In this section we show that the system depicted in Fig. 1 can serve as a source of macroscopic entangled light. We employ the sufficient inseparability criterion derived in [18] to provide evidence for the entanglement of the two field modes.

By definition, the quantum state $\varrho_{\mathrm{F}}$ of the cavity field is said to be entangled if and only if it is nonseparable, and $\varrho_{\mathrm{F}}$ is separable if and only if it can be written as

$$
\varrho_{\mathrm{F}}=\sum_{j} p_{j} \varrho_{j}^{(1)} \otimes \varrho_{j}^{(2)}
$$

Here $\varrho_{j}^{(1)}$ and $\varrho_{j}^{(2)}$ are normalized states of the modes 1 and 2 , respectively, and the parameters $p_{j} \geqslant 0$ comply with $\Sigma_{j} p_{j}$ $=1$. The criterion derived in [18] states that the system is in an entangled quantum state if the total variance of two Einstein-Podolsky-Rosen (EPR) type operators $\hat{u}$ and $\hat{v}$ of the two modes satisfies the inequality 


$$
\left\langle(\Delta \hat{u})^{2}+(\Delta \hat{v})^{2}\right\rangle<2
$$

where

$$
\hat{u}=\hat{x}_{1}+\hat{x}_{2}, \quad \hat{v}=\hat{p}_{1}-\hat{p}_{2} .
$$

Here $\hat{x}_{k}$ and $\hat{p}_{k}$ are local operators which correspond to the mode $k$ with frequency $\nu_{k}$. They must obey the commutation relation

$$
\left[\hat{x}_{k}, \hat{p}_{l}\right]=i \delta_{k l},
$$

but are otherwise arbitrary. For the physical system considered here, it turns out that the quadrature operators

$$
\hat{x}_{k}=\left(b_{k}+b_{k}^{\dagger}\right) / \sqrt{2} \text { and } \hat{p}_{k}=\left(b_{k}-b_{k}^{\dagger}\right) /(\sqrt{2} i)
$$

are the best choice, where

$$
b_{k}(t)=a_{k} \exp \left(i \nu_{k} t\right) \quad \text { and } \quad b_{k}^{\dagger}(t)=a_{k}^{\dagger} \exp \left(-i \nu_{k} t\right) .
$$

With the help of Eqs. (23) and (26), we express the total variance of the operators $\hat{u}$ and $\hat{v}$ in terms of the operators $b_{k}$ and $b_{k}^{\dagger}$,

$$
\begin{aligned}
\left\langle(\Delta \hat{u})^{2}+(\Delta \hat{v})^{2}\right\rangle= & 2\left[1+\left\langle b_{1}^{\dagger} b_{1}\right\rangle+\left\langle b_{2}^{\dagger} b_{2}\right\rangle+\left\langle b_{1} b_{2}\right\rangle+\left\langle b_{1}^{\dagger} b_{2}^{\dagger}\right\rangle\right. \\
& \left.-\left\langle b_{1}\right\rangle\left\langle b_{1}^{\dagger}\right\rangle-\left\langle b_{2}\right\rangle\left\langle b_{2}^{\dagger}\right\rangle-\left\langle b_{1}\right\rangle\left\langle b_{2}\right\rangle-\left\langle b_{1}^{\dagger}\right\rangle\left\langle b_{2}^{\dagger}\right\rangle\right] .
\end{aligned}
$$

In Appendix B, we outline the calculation of the mean values that enter Eq. (27).

Next we classify several parameter regimes for which the inequality (22) is satisfied. In the first step, we consider the case where the Rabi frequency $\left|\Omega_{3}\right|$ and the detuning $\Delta_{b}$ are much larger than the parameters $\left|\Delta_{a}\right|,\left|\Omega_{4}\right|, \Gamma_{i}$ $(i \in\{1,2,3,4\})$, i.e.,

$$
\left|\Omega_{3}\right|, \quad\left|\Delta_{b}\right| \gg\left|\Delta_{a}\right|, \quad\left|\Omega_{4}\right|, \quad \Gamma_{i} .
$$

If these conditions are satisfied, the parameters $\alpha_{i j}$ and $\beta_{i j}$ in Eqs. (A1)-(A8) of Appendix A reduce to

$$
\begin{gathered}
\alpha_{11} \approx 0, \quad \alpha_{22} \approx 0, \quad \beta_{11} \approx 0, \quad \beta_{22} \approx 0, \\
\alpha_{21} \approx 0, \quad \beta_{12} \approx 0, \quad \alpha_{12} \approx \beta_{21} \approx-i \alpha \exp \left[i\left(\phi_{3}+\phi_{4}\right) t\right], \\
\alpha=g_{1} g_{2} \frac{\left|\Omega_{4}\right|}{\left|\Omega_{3}\right| \Delta_{b}} .
\end{gathered}
$$

In these equations, $\phi_{3}$ and $\phi_{4}$ are the phases of the classical laser fields with Rabi frequencies $\Omega_{3}=\left|\Omega_{3}\right| \exp \left(i \phi_{3}\right)$ and $\left|\Omega_{4}\right| \exp \left(i \phi_{4}\right)$, respectively (see Sec. II). If the approximate parameters in Eq. (29) are plugged into Eq. (20), we obtain the equation of motion for the density operator $\varrho_{\mathrm{F}}$ of the cavity modes in the limit (28),

$$
\begin{aligned}
\dot{\varrho}_{\mathrm{F}}= & -i \nu_{1}\left[a_{1}^{\dagger} a_{1}, \varrho_{\mathrm{F}}\right]-i \nu_{2}\left[a_{2}^{\dagger} a_{2}, \varrho_{\mathrm{F}}\right]+i\left[H_{\mathrm{P}}, \varrho_{\mathrm{F}}\right]-\kappa\left(a_{1}^{\dagger} a_{1} \varrho_{\mathrm{F}}\right. \\
& \left.+\varrho_{\mathrm{F}} a_{1}^{\dagger} a_{1}-2 a_{1} \varrho_{\mathrm{F}} a_{1}^{\dagger}+a_{2}^{\dagger} a_{2} \varrho_{\mathrm{F}}+\varrho_{\mathrm{F}} a_{2}^{\dagger} a_{2}-2 a_{2} \varrho_{\mathrm{F}} a_{2}^{\dagger}\right),
\end{aligned}
$$

where

$$
\begin{aligned}
H_{\mathrm{P}}= & \alpha a_{1}^{\dagger} a_{2}^{\dagger} \exp \left[i\left(\phi_{3}+\phi_{4}\right) t\right] \exp \left[-i\left(\nu_{1}+\nu_{2}\right) t\right] \\
& +\alpha a_{1} a_{2} \exp \left[-i\left(\phi_{3}+\phi_{4}\right) t\right] \exp \left[i\left(\nu_{1}+\nu_{2}\right) t\right] .
\end{aligned}
$$

Here we assumed for the sake of simplicity that the decay rates of the cavity modes are equal, $\kappa_{1}=\kappa_{2}=\kappa$. We identify Eq. (30) as the master equation for a nondegenerate parametric oscillator in the parametric approximation [25]. Note that this parametric limit was also obtained in the case of a twomode correlated spontaneous emission laser discussed in [12]. Next we evaluate the total variance of the operators $\hat{u}$ and $\hat{v}$ in Eq. (27) and the mean number of photons $\langle\hat{N}\rangle$ $=\left\langle a_{1}^{\dagger} a_{1}+a_{2}^{\dagger} a_{2}\right\rangle=\left\langle b_{1}^{\dagger} b_{1}+b_{2}^{\dagger} b_{2}\right\rangle$ with the approximate density operator $\varrho_{\mathrm{F}}$ in Eq. (30). If the sum of the laser phases obeys $\phi_{3}+\phi_{4}=\pi / 2$, we obtain [12]

$$
\begin{aligned}
\left\langle(\Delta \hat{u})^{2}+(\Delta \hat{v})^{2}\right\rangle(t) & \left(\left\langle(\Delta \hat{u})^{2}+(\Delta \hat{v})^{2}\right\rangle(0)-\frac{2 \kappa}{\alpha+\kappa}\right) e^{-2(\alpha+\kappa) t}+\frac{2 \kappa}{\alpha+\kappa}, \\
= & (\langle\hat{N}\rangle(t)= \\
& -\left(\frac{\alpha \kappa}{\kappa^{2}-\alpha^{2}}+\left\langle a_{1} a_{2}+a_{1}^{\dagger} a_{2}^{\dagger}\right\rangle(0)\right) \sinh (2 \alpha t) e^{-2 \kappa t} \\
& +\frac{\alpha^{2}}{\kappa^{2}-\alpha^{2}} .
\end{aligned}
$$

It follows from Eq. (32) that the entanglement criterion in Eq. (22) is satisfied for any initial state of the cavity field if $(\alpha+\kappa) t \gg 1$ and $\alpha>0$ [12]. The time evolution of the total variance of the operators $\hat{u}$ and $\hat{v}$ is shown in Fig. 2(a). The dashed curve shows $\left\langle(\Delta \hat{u})^{2}+(\Delta \hat{v})^{2}\right\rangle$ according to Eq. (32), and the solid line corresponds to the general case where the mean values in Eq. (27) are evaluated with the full density operator $\varrho_{\mathrm{F}}$ in Eq. (20). The cavity modes are assumed to be in the vacuum state initially, and the parameters comply with condition (28). It follows from Fig. 2 that the approximate result in Eq. (32) is in good agreement with the exact solution only if $g t<300$. While the light field remains in an entangled state in the parametric case, the exact solution demonstrates that the entanglement of the cavity field exists only for a finite period of time.

Next we discuss the time evolution of the mean number of photons $\langle\hat{N}\rangle$. According to Eq. (33), $\langle\hat{N}\rangle$ grows exponentially with time for any initial state of the cavity field, provided that $(\alpha-\kappa) t \gg 1$ and $\alpha>\kappa[12]$. The time evolution of $\langle\hat{N}\rangle$ is shown in Fig. 2(b) on a logarithmic scale. In contrast to $\left\langle(\Delta \hat{u})^{2}+(\Delta \hat{v})^{2}\right\rangle$, the result for $\langle\hat{N}\rangle$ in the parametric approximation (dashed line) is in good agreement with the exact solution (solid line) even for $g t \gg 300$. Moreover, Fig. 2(b) shows that the mean number of photons grows exponentially if the scaled time $g t$ is sufficiently large.

According to Fig. 2, the entangled state of the cavity field contains up to $\langle\hat{N}\rangle \approx 110$ photons on average. It follows that the single-atom laser depicted in Fig. 1 can give rise to an 


$$
\left\langle(\Delta \hat{u})^{2}+(\Delta \hat{v})^{2}\right\rangle
$$

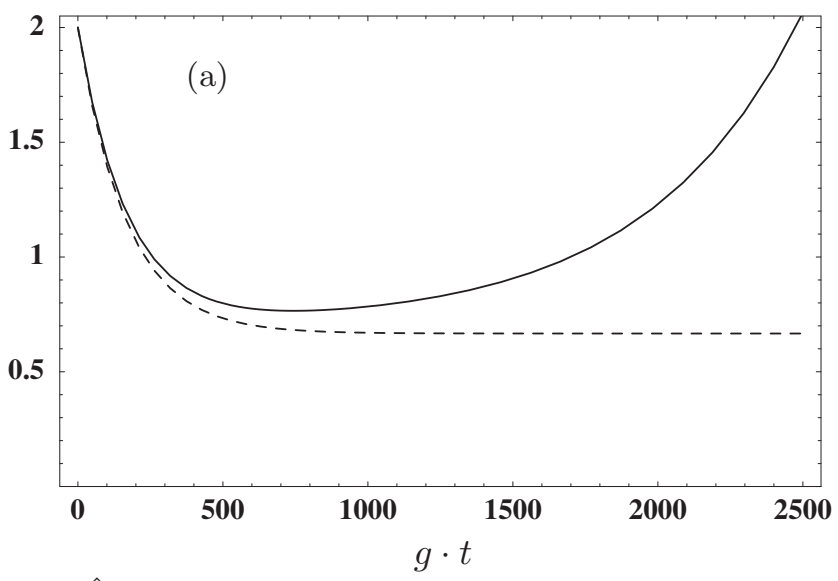

$\langle\hat{N}\rangle$

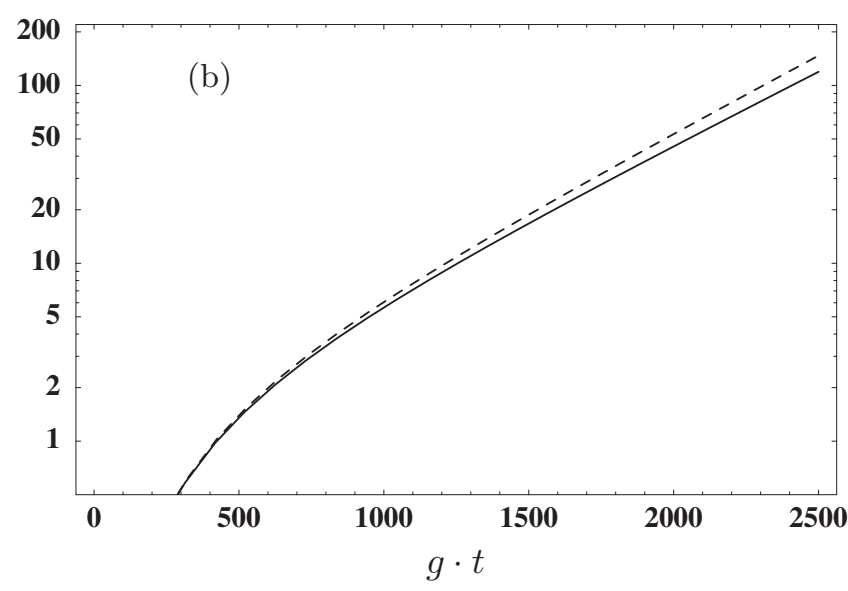

FIG. 2. (a) Time evolution of $\left\langle(\Delta \hat{u})^{2}+(\Delta \hat{v})^{2}\right\rangle$. The mean value of the total number of photons $\langle\hat{N}\rangle$ is shown in (b) on a logarithmic scale. At $t=0$, the cavity field is assumed to be in the vacuum state. The dashed curves were obtained with the density operator of the parametric oscillator in Eq. (30), and the solid curves correspond to the full density operator in Eq. (20). The parameters are $g_{1}=g_{2}=g$, $\left|\Omega_{3}\right|=25 g,\left|\Omega_{4}\right|=2 g, \Gamma_{1}=\Gamma_{2}=\Gamma_{3}=\Gamma_{4}=5 g, \Delta_{a}=0, \Delta_{b}=40 g, \kappa_{1}=\kappa_{2}$ $=10^{-3} \mathrm{~g}$, and $\phi_{3}+\phi_{4}=\pi / 2$.

entangled quantum state of the two cavity modes if the parameters are in agreement with condition (28). If this condition holds, level $|b\rangle$ is almost unexcited due to the large detuning $\Delta_{b}$, and states $|c\rangle$ and $|d\rangle$ are coupled via a twophoton process. In contrast, the transitions $|d\rangle \leftrightarrow|a\rangle$ and $|c\rangle \leftrightarrow|a\rangle$ are driven resonantly. In this situation, the structure of the Hamiltonian $H_{P}$ in Eq. (31) implies that the system can emit photons into the cavity fields only in pairs, where one photon is emitted in mode 1 and the other photon in mode 2 . If the cavity field is initially in the vacuum state $|0,0\rangle$, it will evolve under the influence of $H_{P}$ into the entangled state

$$
a|0,0\rangle+b|1,1\rangle+c|2,2\rangle+\cdots
$$

where $a, b$, and $c$ are complex coefficients. If the complicated master equation (20) can be reduced under certain con- ditions to the parametric equation (30), it is thus clear that a macroscopic entangled state is generated.

Due to the symmetry in the atomic level scheme, it is possible to reverse the role of the transitions $|d\rangle \leftrightarrow|a\rangle \leftrightarrow|c\rangle$ and $|c\rangle \leftrightarrow|b\rangle \leftrightarrow|d\rangle$. In this case, the detuning $\Delta_{a}$ is large and the transitions $|d\rangle \leftrightarrow|b\rangle$ and $|c\rangle \leftrightarrow|b\rangle$ are driven resonantly. Condition (28) then has to be replaced by

$$
\left|\Omega_{4}\right|, \quad\left|\Delta_{a}\right| \gg\left|\Delta_{b}\right|, \quad\left|\Omega_{3}\right|, \quad \Gamma_{i},
$$

and the only nonvanishing coefficients in Eq. (20) are now determined by $\alpha_{21} \approx \beta_{12} \approx-i \alpha^{\prime} \exp \left[i\left(\phi_{3}+\phi_{4}\right) t\right]$, where $\alpha^{\prime}$ $=g_{1} g_{2}\left|\Omega_{3}\right| /\left(\left|\Omega_{4}\right| \Delta_{a}\right)$. It follows that the results in Eqs. (30), (32), and (33) are also valid if condition (35) holds, provided that $\alpha$ is replaced by $\alpha^{\prime}$.

We now demonstrate that it can be advantageous to consider parameters which do not comply with condition (28) or (35). Since the approximate results in Eqs. (32) and (33) do not apply in this case, we evaluate the mean values $\left\langle(\Delta \hat{u})^{2}\right.$ $\left.+(\Delta \hat{v})^{2}\right\rangle$ and $\langle\hat{N}\rangle$ only with the exact density operator $\varrho_{\mathrm{F}}$ in Eq. (20). The time evolution of $\left\langle(\Delta \hat{u})^{2}+(\Delta \hat{v})^{2}\right\rangle$ is shown in Fig. 3(a) for two sets of parameters. As compared to the parameters chosen for Fig. 2, the magnitude of the Rabi frequency $\Omega_{4}$ has been increased such that $\left|\Omega_{3}\right|$ is still larger, but not much larger than $\left|\Omega_{4}\right|$. It follows from Fig. 3(a) that the entanglement criterion in Eq. (22) is satisfied for shorter times as compared to the solid line in Fig. 2(a). On the other hand, Fig. 3(b) shows that the mean number of photons can be much larger as compared to Fig. 2(b). For curve I of Fig. 3(a), the maximum mean number of photons for which the entanglement criterion (22) is still satisfied is $\langle\hat{N}\rangle \approx 10.2$ $\times 10^{4}$. The same number for the parameters of curve II reads $\langle\hat{N}\rangle \approx 6100$. As compared to Fig. 2 , the maximum mean number of photons can be enhanced by several orders of magnitude.

Finally, we consider the case where the quantum state of the cavity field is initially the coherent state $|100,-100\rangle$. The time evolution of $\left\langle(\Delta \hat{u})^{2}+(\Delta \hat{v})^{2}\right\rangle$ and $\langle\hat{N}\rangle$ is shown in Fig. 4 for two sets of parameters. All mean values were evaluated with the exact density operator in Eq. (20). For curve I, the magnitude of the Rabi frequency $\Omega_{4}$ is larger as compared to curve II. All other parameters are the same for curve I and II. It can be seen from Fig. 4(a) that the entanglement criterion is satisfied for shorter times if $\left|\Omega_{4}\right|$ is increased. In contrast, the mean number of photons can be greatly enhanced if the value of $\left|\Omega_{4}\right|$ is increased, as can be seen from Fig. 4(b). Similar conclusions can be drawn from Fig. 3, where the initial state of the cavity field is the vacuum. The comparison of Figs. 3 and 4 shows that the mean number of photons can be much larger than in Fig. 3 if the cavity field is initially prepared in a coherent state. Due to the large mean number of photons in the cavity modes, the system may leave the regime of linear amplification such that saturation effects modify the curve progression in Fig. 4. These effects are described by terms that go beyond the second-order expansion of the atom-cavity coupling and are neglected here. According to the linear theory, the maximum mean number of photons for which the entanglement criterion (22) is still 


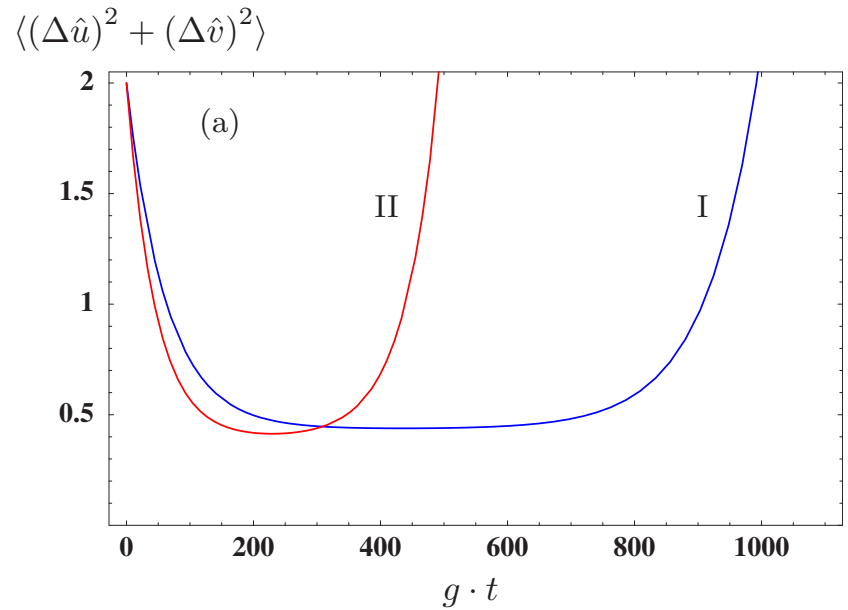

$\langle\hat{N}\rangle$

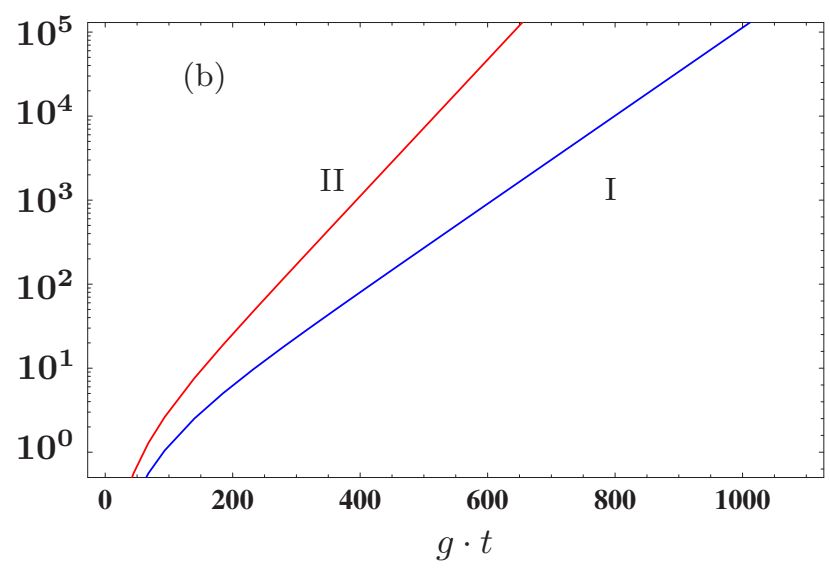

FIG. 3. (Color online) (a) Time evolution of $\left\langle(\Delta \hat{u})^{2}+(\Delta \hat{v})^{2}\right\rangle$. The mean value of the total number of photons $\langle\hat{N}\rangle$ is shown in (b) on a logarithmic scale. At $t=0$, the cavity field is assumed to be in the vacuum state, and we set $\Gamma_{1}=\Gamma_{2}=\Gamma_{3}=\Gamma_{4}=5 g, g_{1}=g_{2}=g, \kappa_{1}$ $=\kappa_{2}=10^{-3} \mathrm{~g}$ and $\phi_{3}+\phi_{4}=\pi / 2$. The parameters for the curves labeled I are $\left|\Omega_{3}\right|=25 g,\left|\Omega_{4}\right|=9.8 g, \Delta_{a}=0, \Delta_{b}=43 g$, and for II we have $\left|\Omega_{3}\right|=15 g,\left|\Omega_{4}\right|=6 g, \Delta_{a}=0, \Delta_{b}=32.5 g$.

satisfied is $\langle\hat{N}\rangle \approx 6.5 \times 10^{5}$ in the case of curve II of Fig. 4 , and in the case of curve I the entangled cavity field contains up to $\langle\hat{N}\rangle \approx 5.4 \times 10^{7}$ photons.

\section{CONCLUSION}

We have shown that a two-mode single-atom laser can serve as a source of macroscopic entangled light. We identified two parameter regimes for which the quantum state of the cavity field is entangled for a long period of time. For these parameters, the master equation for the density operator of the two cavity modes can be approximately reduced to the master equation for a nondegenerate parametric oscillator in the parametric approximation.

The mean number of photons in the cavity field can be strongly increased if parameters beyond the parametric limit are chosen. This enhancement of the mean photon numbers is accompanied by a shortening of the time slice for which the entanglement criterion is satisfied. As the initial state of
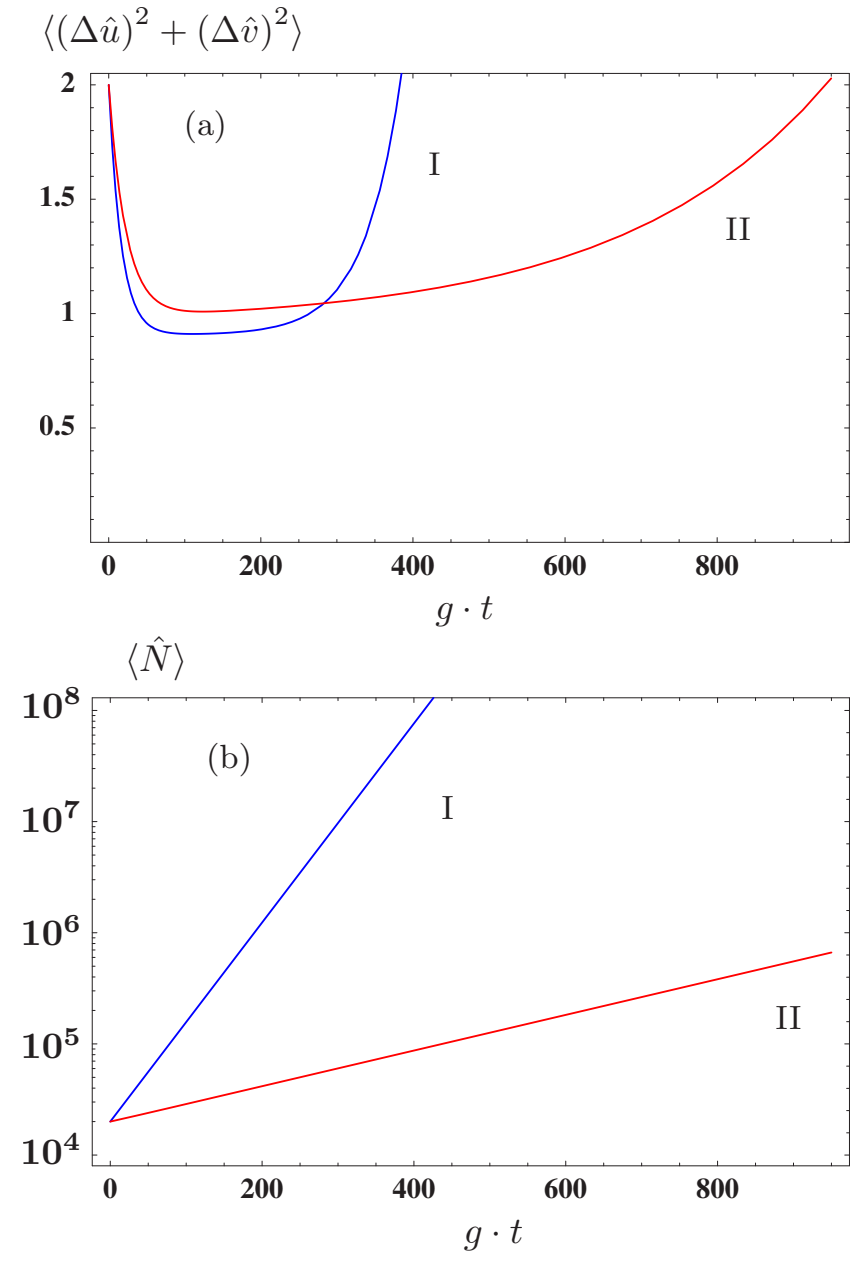

FIG. 4. (Color online) (a) Time evolution of $\left\langle(\Delta \hat{u})^{2}+(\Delta \hat{v})^{2}\right\rangle$. The mean value of the total number of photons $\langle\hat{N}\rangle$ is shown in (b) on a logarithmic scale. At $t=0$, the cavity field is assumed to be in the coherent state $|100,-100\rangle$, and we set $\Gamma_{1}=\Gamma_{2}=\Gamma_{3}=\Gamma_{4}=2 g, g_{1}$ $=g_{2}=g, \kappa_{1}=\kappa_{2}=10^{-2} g$, and $\phi_{3}+\phi_{4}=\pi / 2$. The parameters for the curves labeled I are $\left|\Omega_{3}\right|=10 g,\left|\Omega_{4}\right|=5 g, \Delta_{a}=0, \Delta_{b}=15 g$, and for II we have $\left|\Omega_{3}\right|=10 g,\left|\Omega_{4}\right|=2 g, \Delta_{a}=0, \Delta_{b}=15 g$.

the cavity field, we chose either the vacuum or a coherent state. We demonstrated that the mean number of photons of the entangled cavity field can increase by several orders of magnitude if a coherent state instead of the vacuum is chosen as the initial state.

\section{APPENDIX A: COEFFICIENTS}

Here we give the explicit definitions of the coefficients $\alpha_{i j}$ and $\beta_{i j}$ which enter the master equation (20) for the density operator $\varrho_{\mathrm{F}}$ of the two cavity modes:

$$
\begin{aligned}
& \alpha_{11}=2 g_{1}^{2} \Gamma_{2}\left|\Omega_{3}\right|^{2}\left|\Omega_{4}\right|^{2}\left\{4\left(P_{2}^{*}+4 i \Delta_{b}\right)\left|\Omega_{4}\right|^{2}+P_{1}^{*}\left[4\left|\Omega_{3}\right|^{2}\right.\right. \\
&+\left.\left.P_{1}\left(P_{1}+P_{2}^{*}\right)\right]\right\} /\left(P_{3} P_{4}\right), \\
& \beta_{11}=-2 g_{1}^{2} \Gamma_{4}\left|\Omega_{3}\right|^{2}\left|\Omega_{4}\right|^{2}\left\{4 P_{1}\left|\Omega_{4}\right|^{2}+P_{2}^{*}\left[4\left|\Omega_{3}\right|^{2}\right.\right. \\
&\left.\left.+P_{1}\left(P_{1}+P_{2}^{*}\right)\right]\right\} /\left(P_{3} P_{4}\right),
\end{aligned}
$$




$$
\begin{aligned}
\alpha_{12}= & -2 g_{1} g_{2} \Gamma_{2} \Omega_{3} \Omega_{4}\left|\Omega_{3}\right|^{2}\left[4 P_{1}\left|\Omega_{3}\right|^{2}+P_{1}^{2}\left(P_{1}+P_{2}^{*}\right)\right. \\
& \left.-4\left|\Omega_{4}\right|^{2}\left(2 P_{1}+P_{2}^{*}\right)\right] /\left(P_{3} P_{4}\right) \\
\beta_{12}= & -2 g_{1} g_{2} \Gamma_{4} \Omega_{3} \Omega_{4}\left|\Omega_{4}\right|^{2}\left[\left(P_{1}+P_{2}^{*}\right)\left|P_{2}\right|^{2}+4\left|\Omega_{4}\right|^{2} P_{2}\right. \\
& \left.+4\left|\Omega_{3}\right|^{2}\left(P_{1}-4 i \Delta_{a}\right)\right] /\left(P_{3} P_{4}\right) \\
\alpha_{22}= & 2 g_{2}^{2} \Gamma_{4}\left|\Omega_{3}\right|^{2}\left|\Omega_{4}\right|^{2}\left\{4\left(P_{1}^{*}+4 i \Delta_{a}\right)\left|\Omega_{3}\right|^{2}+P_{2}^{*}\left[4\left|\Omega_{4}\right|^{2}\right.\right. \\
& \left.\left.+P_{2}\left(P_{2}+P_{1}^{*}\right)\right]\right\} /\left(P_{3} P_{5}\right), \\
\beta_{22}= & -2 g_{2}^{2} \Gamma_{2}\left|\Omega_{3}\right|^{2}\left|\Omega_{4}\right|^{2}\left\{4 P_{2}\left|\Omega_{3}\right|^{2}+P_{1}^{*}\left[4\left|\Omega_{4}\right|^{2}\right.\right. \\
& \left.\left.+P_{2}\left(P_{2}+P_{1}^{*}\right)\right]\right\} /\left(P_{3} P_{5}\right), \\
\alpha_{21}= & -2 g_{1} g_{2} \Gamma_{4} \Omega_{3} \Omega_{4}\left|\Omega_{4}\right|^{2}\left[4 P_{2}\left|\Omega_{4}\right|^{2}+P_{2}^{2}\left(P_{2}+P_{1}^{*}\right)\right. \\
& \left.-4\left|\Omega_{3}\right|^{2}\left(2 P_{2}+P_{1}^{*}\right)\right] /\left(P_{3} P_{5}\right), \\
\beta_{21}= & -2 g_{1} g_{2} \Gamma_{2} \Omega_{3} \Omega_{4}\left|\Omega_{3}\right|^{2}\left[\left(P_{2}+P_{1}^{*}\right)\left|P_{1}\right|^{2}+4\left|\Omega_{3}\right|^{2} P_{1}\right. \\
+ & \left.4\left|\Omega_{4}\right|^{2}\left(P_{2}-4 i \Delta_{b}\right)\right] /\left(P_{3} P_{5}\right) .
\end{aligned}
$$

The parameters $P_{1}, P_{2}, P_{3}, P_{4}$, and $P_{5}$ in Eqs. (A1)-(A8) are defined as

$$
\begin{gathered}
P_{1}=\Gamma_{3}+\Gamma_{4}+2 i \Delta_{b}, \\
P_{2}=\Gamma_{1}+\Gamma_{2}+2 i \Delta_{a}, \\
P_{3}=\Gamma_{2}\left|P_{1}\right|^{2}\left|\Omega_{3}\right|^{2}+\Gamma_{4}\left|P_{2}\right|^{2}\left|\Omega_{4}\right|^{2}+8\left|\Omega_{3}\right|^{2}\left|\Omega_{4}\right|^{2}\left(\Gamma_{2}+\Gamma_{4}\right),
\end{gathered}
$$

$P_{4}=4\left[\left|\Omega_{3}\right|^{2}-\left|\Omega_{4}\right|^{2}\right]^{2}+P_{1}\left(P_{1}+P_{2}^{*}\right)\left|\Omega_{3}\right|^{2}+P_{2}^{*}\left(P_{1}+P_{2}^{*}\right)\left|\Omega_{4}\right|^{2}$,

$P_{5}=4\left[\left|\Omega_{3}\right|^{2}-\left|\Omega_{4}\right|^{2}\right]^{2}+P_{1}^{*}\left(P_{2}+P_{1}^{*}\right)\left|\Omega_{3}\right|^{2}+P_{2}\left(P_{2}+P_{1}^{*}\right)\left|\Omega_{4}\right|^{2}$.

\section{APPENDIX B: CALCULATION OF THE MEAN VALUES}

In the following, we outline the calculation of the mean values that enter the total variance of the operators $\hat{u}$ and $\hat{v}$ in Eq. (27). We begin with the mean values of the quadrature operators defined in Eq. (25) with respect to the density operator $\varrho_{\mathrm{F}}$ of the two cavity modes. With the help of Eq. (20), we derive the following system of differential equations for the mean values $\left\langle b_{1}\right\rangle$ and $\left\langle b_{2}^{\dagger}\right\rangle$ :

$$
\partial_{t}\left(\begin{array}{c}
\left\langle b_{1}\right\rangle \\
\left\langle b_{2}^{\dagger}\right\rangle
\end{array}\right)=-\left(\begin{array}{cc}
C_{11}+\kappa_{1} & C_{12} \\
C_{21}^{*} & C_{22}^{*}+\kappa_{2}
\end{array}\right)\left(\begin{array}{c}
\left\langle b_{1}\right\rangle \\
\left\langle b_{2}^{\dagger}\right\rangle
\end{array}\right),
$$

and $C_{i j}=\alpha_{i j}+\beta_{i j}$. The solution to this set of coupled equations is

$$
\begin{aligned}
\left\langle b_{1}\right\rangle= & e^{w_{2} t}\left(\cosh \left(w_{1} t\right)\left\langle b_{1}\right\rangle_{0}+\frac{1}{2 w_{1}}\right. \\
& \left.\times\left[\left\langle b_{1}\right\rangle_{0}\left(C_{22}^{*}-C_{11}-\kappa_{1}+\kappa_{2}\right)-2\left\langle b_{2}^{\dagger}\right\rangle_{0} C_{12}\right] \sinh \left(w_{1} t\right)\right), \\
\left\langle b_{2}^{\dagger}\right\rangle= & e^{w_{2} t}\left(\cosh \left(w_{1} t\right)\left\langle b_{2}^{\dagger}\right\rangle_{0}+\frac{1}{2 w_{1}}\right. \\
& \left.\times\left[\left\langle b_{2}^{\dagger}\right\rangle_{0}\left(C_{11}-C_{22}^{*}+\kappa_{1}-\kappa_{2}\right)-2\left\langle b_{1}\right\rangle_{0} C_{21}^{*}\right] \sinh \left(w_{1} t\right)\right),
\end{aligned}
$$

where

$$
\begin{gathered}
w_{1}=\frac{1}{2} \sqrt{4 C_{12} C_{21}^{*}+\left(C_{11}-C_{22}^{*}+\kappa_{1}-\kappa_{2}\right)^{2}}, \\
w_{2}=-\frac{1}{2}\left(C_{11}+C_{22}^{*}+\kappa_{1}+\kappa_{2}\right),
\end{gathered}
$$

and $\langle\cdot\rangle_{0}=\langle\cdot\rangle(t=0)$ denotes the initial mean value at $t=0$. Note that the mean values $\left\langle b_{1}^{\dagger}\right\rangle$ and $\left\langle b_{2}\right\rangle$ can be obtained from $\left\langle b_{1}\right\rangle$ and $\left\langle b_{2}^{\dagger}\right\rangle$ by complex conjugation, i.e., $\left\langle b_{1}^{\dagger}\right\rangle=\left\langle b_{1}\right\rangle^{*}$ and $\left\langle b_{2}\right\rangle$ $=\left\langle b_{2}^{\dagger}\right\rangle^{*}$.

The remaining mean values in Eq. (27) involve products of the operators $b_{i}$ and $b_{i}^{\dagger}$. With the aid of Eq. (20), we obtain the following set of differential equations:

$$
\partial_{t} \boldsymbol{R}=M \boldsymbol{R}+\boldsymbol{I}
$$

where $\boldsymbol{R}=\left(\left\langle b_{1}^{\dagger} b_{1}\right\rangle,\left\langle b_{2}^{\dagger} b_{2}\right\rangle,\left\langle b_{1} b_{2}\right\rangle,\left\langle b_{1}^{\dagger} b_{2}^{\dagger}\right\rangle\right)$ and

$$
M=-\left(\begin{array}{cccc}
D_{11} & 0 & C_{12}^{*} & C_{12} \\
0 & D_{22} & C_{21}^{*} & C_{21} \\
C_{21} & C_{12} & D_{12} & 0 \\
C_{21}^{*} & C_{12}^{*} & 0 & D_{12}^{*}
\end{array}\right), \quad I=-\left(\begin{array}{c}
\beta_{11}+\beta_{11}^{*} \\
\beta_{22}+\beta_{22}^{*} \\
\alpha_{12}+\alpha_{21} \\
\alpha_{12}^{*}+\alpha_{21}^{*}
\end{array}\right) .
$$

The elements of the matrix $M$ are defined as

$$
\begin{gathered}
C_{i j}=\alpha_{i j}+\beta_{i j}, \quad D_{i i}=\alpha_{i i}+\alpha_{i i}^{*}+\beta_{i i}+\beta_{i i}^{*}+2 \kappa_{i}, \\
D_{12}=C_{11}+C_{22}+\kappa_{1}+\kappa_{2} .
\end{gathered}
$$

The differential equation (B5) can be solved numerically without difficulties. An analytical solution can be obtained, for example, by means of the Laplace transform method, which yields the following results for the components $R_{i}$ of the vector $\boldsymbol{R}$ :

$$
R_{i}=\sum_{k=1}^{4}\left[\operatorname{Res}\left(f_{i}, \lambda_{k}\right)+\operatorname{Res}\left(g_{i}, \lambda_{k}\right)\right] e^{\lambda_{k} t}+\operatorname{Res}\left(g_{i}, 0\right) .
$$

In this equation, expressions of the type $\operatorname{Res}(h, z)$ denote the residue of the function $h$ evaluated at $z$, and the functions $f$ $=\left(f_{1}, f_{2}, f_{3}, f_{4}\right)$ and $\boldsymbol{g}=\left(g_{1}, g_{2}, g_{3}, g_{4}\right)$ are determined by 


$$
\boldsymbol{f}(s)=\left[s \hat{1}_{4}-M\right]^{-1} \boldsymbol{R}_{0} \quad \text { and } \quad \boldsymbol{g}(s)=\left[s \hat{1}_{4}-M\right]^{-1}(\boldsymbol{I} / s),
$$

respectively. Here $\hat{1}_{4}$ denotes the $4 \times 4$ identity matrix, and the vector $\boldsymbol{R}_{0}$ is the initial value of $\boldsymbol{R}$ at $t=0, \boldsymbol{R}_{0}$ $=\left(\left\langle b_{1}^{\dagger} b_{1}\right\rangle_{0},\left\langle b_{2}^{\dagger} b_{2}\right\rangle_{0},\left\langle b_{1} b_{2}\right\rangle_{0},\left\langle b_{1}^{\dagger} b_{2}^{\dagger}\right\rangle_{0}\right)$. Finally, the parameters $\lambda_{k}$ are the four (complex) eigenvalues of the matrix $M$ which is defined in Eq. (B6). The eigenvalues $\lambda_{k}$ can be obtained as the roots of the following equation:

$$
\begin{aligned}
s^{4}+ & \left(D_{11}+D_{22}+D_{12}+D_{12}^{*}\right) s^{3}+\left[\left|D_{12}\right|^{2}-2 C_{21} C_{12}^{*}-2 C_{12} C_{21}^{*}\right. \\
& \left.+D_{11} D_{22}+\left(D_{11}+D_{22}\right)\left(D_{12}+D_{12}^{*}\right)\right] s^{2} \\
& +\left[\left(D_{11}+D_{22}\right)\left|D_{12}\right|^{2}-\left(C_{21} C_{12}^{*}+C_{12} C_{21}^{*}\right)\left(D_{11}+D_{22}\right.\right. \\
& \left.\left.+D_{12}+D_{12}^{*}\right)+D_{11} D_{22}\left(D_{12}+D_{12}^{*}\right)\right] s+C_{21}^{2}\left(C_{12}^{*}\right)^{2} \\
& -\left(2 C_{12} C_{21}^{*}+D_{11} D_{12}+D_{22} D_{12}^{*}\right) C_{21} C_{12}^{*}+\left(C_{12} C_{21}^{*}\right. \\
& \left.-D_{22} D_{12}\right)\left(C_{12} C_{21}^{*}-D_{11} D_{12}^{*}\right)=0 .
\end{aligned}
$$

[1] M. A. Nielsen and I. L. Chuang, Quantum Computation and Quantum Information (Cambridge University Press, Cambridge, U.K., 2000).

[2] C. H. Bennett, G. Brassard, C. Crepeau, R. Jozsa, A. Peres, and W. K. Wootters, Phys. Rev. Lett. 70, 1895 (1993).

[3] D. Bouwmeester, J. W. Pan, K. Mattle, M. Eibl, H. Weinfurter, and A. Zeilinger, Nature (London) 390, 575 (1997).

[4] C. H. Bennett and G. Brassard, in Proceedings of the IEEE International Conference on Computers, Systems and Signal Processing (IEEE Computer Society Press, Los Alamitos, CA, 1984), p. 175.

[5] P. W. Shor, in Proceedings of the 35th Annual Symposium on Foundations of Computer Science, Santa Fe, New Mexico, 1994, edited by S. Goldwasser (IEEE Computer Society Press, Los Alamitos, CA, 1994), p. 124; SIAM J. Comput. 26, 1484 (1997).

[6] L. K. Grover, Phys. Rev. Lett. 79, 325 (1997).

[7] S. L. Braunstein and P. van Look, Rev. Mod. Phys. 77, 513 (2005).

[8] Z. Y. Ou, S. F. Pereira, H. J. Kimble, and K. C. Peng, Phys. Rev. Lett. 68, 3663 (1992).

[9] G. Morigi, J. Eschner, S. Mancini, and D. Vitali, Phys. Rev. Lett. 96, 023601 (2006).

[10] G. Morigi, J. Eschner, S. Mancini, and D. Vitali, Phys. Rev. A 73, 033822 (2006).
[11] L. Zhou, H. Xiong, and M. S. Zubairy, Phys. Rev. A 74, 022321 (2006)

[12] H. Xiong, M. O. Scully, and M. S. Zubairy, Phys. Rev. Lett. 94, 023601 (2005).

[13] H.-T. Tan, S.-Y. Zhu, and M. S. Zubairy, Phys. Rev. A 72, 022305 (2005).

[14] M. O. Scully, Phys. Rev. Lett. 55, 2802 (1985).

[15] M. O. Scully and M. S. Zubairy, Phys. Rev. A 35, 752 (1987).

[16] J. McKeever, A. Boca, A. D. Boozer, J. R. Buck, and H. J. Kimble, Nature (London) 425, 268 (2003).

[17] R. Simon, Phys. Rev. Lett. 84, 2726 (2000).

[18] L.-M. Duan, G. Giedke, J. I. Cirac, and P. Zoller, Phys. Rev. Lett. 84, 2722 (2000).

[19] E. Shchukin and W. Vogel, Phys. Rev. Lett. 95, 230502 (2005).

[20] M. Hillery and M. S. Zubairy, Phys. Rev. Lett. 96, 050503 (2006).

[21] G. S. Agarwal and A. Biswas, New J. Phys. 7, 211 (2005).

[22] H. Nha and J. Kim, Phys. Rev. A 74, 012317 (2006).

[23] O. Gühne and N. Lütkenhaus, Phys. Rev. Lett. 96, 170502 (2006).

[24] V. Giovannetti, S. Mancini, D. Vitali, and P. Tombesi, Phys. Rev. A 67, 022320 (2003).

[25] M. O. Scully and M. S. Zubairy, Quantum Optics (Cambridge University Press, Cambridge, U.K., 1997). 